\title{
WEAK AMENABILITY OF FOURIER ALGEBRAS ON COMPACT GROUPS
}

\author{
BRIAN E. FORREST, EBRAHIM SAMEI AND NICO SPRONK
}

\begin{abstract}
We give for a compact group $G$, a full characterisation of when its Fourier algebra $\mathrm{A}(G)$ is weakly amenable: when the connected component of the identity $G_{e}$ is abelian. This condition is also equivalent to the hyper-Tauberian property for $\mathrm{A}(G)$, and to having the anti-diagonal $\check{\Delta}=\left\{\left(s, s^{-1}\right): s \in G\right\}$ being a set of spectral synthesis for $\mathrm{A}(G \times G)$. We show the relationship between amenability and weak amenability of $\mathrm{A}(G)$, and (operator) amenability and (operator) weak amenability of $\mathrm{A}_{\Delta}(G)$, an algebra defined by the authors in [10]. We close by extending our results to some classes of non-compact, locally compact groups, including small invariant neighbourhood groups and maximally weakly almost periodic groups.
\end{abstract}

\section{BACKGRound AND Notation}

1.1. Context. B. E. Johnson [22], showed that for the compact Lie group $G=\mathrm{SO}(3)$, the Fourier algebra $\mathrm{A}(G)$ is not weakly amenable. At the time that article was written, the result was a surprise, since it was expected that $\mathrm{A}(G)$ should be amenable for any amenable locally compact group, and in particular for any compact group, in analogy to the famous characterisation of amenability of group algebras $\mathrm{L}^{1}(G)[20$. Very soon after that, Z.-J. Ruan [29] showed that if one puts a suitable operator space structure on $\mathrm{A}(G)$, then $\mathrm{A}(G)$ is operator amenable exactly when $G$ is amenable. This was the first evidence of the important role that the operator space structure would play in understanding the cohomology of $\mathrm{A}(G)$ for non-commuative groups. Indeed, Johnson's paper combined with earlier work of V. Losert [25], led to the conjecture that $\mathrm{A}(G)$ would be amenable as a Banach algebra precisely when $G$ was almost abelian, i.e. $G$ admits an abelian subgroup of finite index. This conjecture was finally settled affirmatively by the first author and V. Runde [9]. Refelected in this result is the fact that the Fourier algebra

Date: October 25, 2018.

2000 Mathematics Subject Classification. Primary 43A30, 43A77, 46M20; Secondary 47L25, 46J10. Key words and phrases. Fourier algebra, weak amenability, spectral synthesis.

Research of the the first named author supported by NSERC Grant 90749-00. Research of the second named author supported by an NSERC Post Doctoral Fellowship. Research of the the third named author supported by NSERC Grant 312515-05. 
has a trivial operator space structure precisely when $G$ admits an abelian subgroup of finite index [12].

On the separate question of weak amenability, Johnson 21] showed that $\mathrm{L}^{1}(G)$ is always weakly amenable. The operator space analogue of that result was established for Fourier algebras by the third author [33, and independently by the second author [31]: $\mathrm{A}(G)$ is always operator weakly amenable. However, the failure of $\mathrm{A}(\mathrm{SO}(3))$ to be weakly amenable in the classical sense demonstrates once again that the operator space category was the right one to consider for weak amenability of $\mathrm{A}(G)$, just as it was for amenability of $\mathrm{A}(G)$. This time though, classical weak amenability for $\mathrm{A}(G)$ did not require the group $G$ itself to be almost abelian. Indeed, as early as 1988, the first author showed $\mathrm{A}(G)$ to be weakly amenable for all discrete $G$. In [22, Johnson proved that $\mathrm{A}(G)$ is weakly amenable for any compact totally disconnected group. This result was extended to a wider class of locally compact groups by the first author in [8]: if $G=A \times D$ where $A$ is abelian and $D$ is totally disconnected, then $\mathrm{A}(G)$ is weakly amenable. Together, these positive results pointed to the structure of the connected component $G_{e}$ of $G$ as the deciding factor in the question of the potential weak amenability of $\mathrm{A}(G)$. In fact, as early as 1996, and even prior to the appearance of [8] in print, it had been conjectured that $\mathrm{A}(G)$ is weakly amenable precisely when $G_{e}$ is abelian. One direction of this conjecture was established by Forrest and Runde [9] who showed that in fact $\mathrm{A}(G)$ is weakly amenable when $G_{e}$, the connected component of the identity in $G$ is abelian. They also presented evidence for the converse when $G$ is a small invariant neighborhood group.

The third author [32] developed the theory of hyper-Tauberian commutative Banach algebras. Though the hyper-Tauberian propety was origianally developed to study reflexivity of spaces of derivations it implies weak amenability, and its theory parallels the theory of weak amenability quite closely. In particular, $\mathrm{A}(G)$ is always operator hyper-Tauberian, and is hyper-Tauberian when $G_{e}$ is abelian. (Unfortunatly, the proof of the latter fact [32, Theorem 21] contains a minor error; this error is repaired in [10, Theorem 3.7].) As is the case with weak amenability, it is thought likely that $\mathrm{A}(G)$ is hyper-Tauberian only when $G_{e}$ is abelian.

In the present article, we show that for compact groups, weak amenability of $\mathrm{A}(G)$ does indeed imply that $G_{e}$ is abelian. Using this result, we can establish the converse of the above mentioned weak amenability conjecture for all small inavriant neighborhood groups and for any maximally almost periodic group. Moreover, our result also shows that many non-abelian, non-compact connected Lie groups do not have weakly amenable Fourier algebras. A similar statement regarding when $\mathrm{A}(G)$ is hyper-Tauberian necessarily follows as well.

The paper is organised as follows. We first deal with compact $G$. In Section 2.1 we establish the main result of this article: $\mathrm{A}(G)$ is weakly amenable only when $G_{e}$ is abelian. Moreover, we show that if $\mathrm{A}(G)$ admits even a 
weakly amenable ideal, then $G_{e}$ must be abelian. In Section 2.2 we show that there is a connection between weak amenability of $\mathrm{A}(G)$ and spectral properties of the antidiagonal $\breve{\Delta}$ for $\mathrm{A}(G \times G)$. We then examine the weak amenability, and amenability of $\mathrm{A}(G)$, in relation to the same properties for $\mathrm{A}_{\Delta}(G)$, in Section 2.3 . The interesting algebras $\mathrm{A}_{\Delta}(G)$ arose in the authors' previous paper [10], and, as we further demonstrate here, hold much of the structural information of the Fourier algebras of compact groups. In the second part of the paper, we use the results for compact groups to obtain similar results for more general locally compact groups, including small invariant neighbourhood groups and maximally almost periodic groups.

The present study owes much of its motivation to work done by the authors in [10], to an extend that we consider this article a successor to that. The second two authors were also motivated by techniques they discovered whilst working on [1. Perhaps not surprisingly, operator spaces will again play a significant role in this investigation.

\subsection{Amenability, weak amenability and the hyper-Tauberian prop-} erty. We will be making non-trivial use of operator spaces in Sections 2.3 and 3.2. We will follow the definitions and notational conventions of [5. In particular, we use the ideas of completely bounded, completely contractive, completey isometric and complete quotient operators; operator dual spaces; and the operator projective tensor product $\mathcal{V} \widehat{\otimes} \mathcal{W}$ of two complete operator spaces. We use $\mathcal{X} \otimes^{\gamma} \mathcal{Y}$ to denote the projective tensor product of two Banach spaces. A completely contractive Banach algebra is a complete operator space $\mathcal{A}$, which admits an associative product $m_{\mathcal{A}}^{0}: \mathcal{A} \otimes \mathcal{A} \rightarrow \mathcal{A}$ which extends to a complete contraction $m_{\mathcal{A}}: \mathcal{A} \widehat{\otimes} \mathcal{A} \rightarrow \mathcal{A}$. A complete operator space and $\mathcal{A}$-bimodule $\mathcal{V}$ is a completely contractive $\mathcal{A}$-bimodule if the left and right actions $\mathcal{A} \otimes \mathcal{V}, \mathcal{V} \otimes \mathcal{A} \rightarrow \mathcal{V}$ extend to complete contractions $\mathcal{A} \widehat{\otimes} \mathcal{V}, \mathcal{V} \widehat{\otimes} \mathcal{A} \rightarrow \mathcal{V}$

Let $\mathcal{A}$ be a (completely contractive) Banach algebra. Following [20, 29], we say that $\mathcal{A}$ is (operator) amenable if every (completely) bounded derivation $D: \mathcal{A} \rightarrow \mathcal{V}^{*}$ - i.e. linear map for which $D(a b)=a \cdot D(b)+D(a) \cdot b$ - for an (completely) bounded dual bimodule $\mathcal{V}$, is inner - i.e. there is $f$ in $\mathcal{V}^{*}$ such that $D a=a \cdot f-f \cdot a$. We say $\mathcal{A}$ is (operator) weakly amenable if every (completely) bounded derivation $D: \mathcal{A} \rightarrow \mathcal{A}^{*}$ is inner. If $\mathcal{A}$ is commutative, (operator) weak amenability is equivalent to having no non-zero derivations $D: \mathcal{A} \rightarrow \mathcal{V}^{*}$ for every symmetric - i.e. $a \cdot v=v \cdot a-$ (completely) bounded bimodule $\mathcal{V}$ (see [2, Theorem 1.5] and [12, Proposition 3.2]).

Let $\mathcal{A}$ be a commutative (completely contractive) Banach algebra. We suppose further that $\mathcal{A}$ is a regular function algebra on a locally compact space $X$. If $\varphi \in \mathcal{A}^{*}$ we define

$$
\operatorname{supp} \varphi=\left\{x \in X: \begin{array}{r}
\text { for every neighbourhood } U \text { of } x \text { there is } f \\
\text { in } \mathcal{A} \text { such that } \operatorname{supp} f \subset U \text { and } \varphi(f) \neq 0
\end{array}\right\} .
$$


Here $\operatorname{supp} f=\overline{\{x \in X: f(x) \neq 0\}}$. An operator $T: \mathcal{A} \rightarrow \mathcal{A}^{*}$ is called a local map if

$$
\operatorname{supp} T u \subset \operatorname{supp} u
$$

for every $u$ in $\mathcal{A}$. We say $\mathcal{A}$ is (operator) hyper-Tauberian on $X$ if every (completely bounded) bounded local map $T: \mathcal{A} \rightarrow \mathcal{A}^{*}$ is an $\mathcal{A}$-module map. This concept was developed by the third author in [32. In that article, all results were stated for the case that $X$ is the Gelfand spectrum of $\mathcal{A}$; however, many of the proofs are valid in the more general assumptions that $\mathcal{A}$ is a regular function algebra on $X$. For example, if $\mathcal{A}$ is (operator) hyper-Tauberian then it is Tauberian and (operator) weakly amenable by [32, Theorem 5] (resp. [32, Theorem 26(ii)]). However, it is possible for $\mathcal{A}$ to be weakly amenable but not hyper-Tauberian [32, Remark 24(ii)].

Now we suppose that $\mathcal{A}$ is a regular (completely contractive) Banach function algebra on a locally compact space $X$. If $E$ is a closed subset of $X$ we let

$$
\begin{aligned}
& \mathrm{I}_{\mathcal{A}}(E)=\{u \in \mathcal{A}: u(x)=0 \text { for every } x \text { in } E\} \\
& \mathrm{I}_{\mathcal{A}}^{c}(E)=\left\{u \in \mathrm{I}_{\mathcal{A}}(E): \operatorname{supp} u \text { is compact }\right\}, \text { and } \\
& \mathrm{I}_{\mathcal{A}}^{0}(E)=\{u \in \mathcal{A}: \operatorname{supp} u \text { is compact and } \operatorname{supp} u \cap E=\varnothing\}
\end{aligned}
$$

We say that $E$ is a set of spectral synthesis, or is simply spectral, for $\mathcal{A}$, if $\mathrm{I}_{\mathcal{A}}(E)=\overline{\mathrm{I}_{\mathcal{A}}^{0}(E)}$. We say $\mathcal{A}$ is Tauberian on $X$ if the empty set $\varnothing$, qua subset of $X$, is a spectral for $\mathcal{A}$. We say $E$ is a set of local synthesis if $\mathrm{I}_{\mathcal{A}}^{c}(E) \subset \overline{\mathrm{I}_{\mathcal{A}}^{0}(E)}$. Note that if $\mathcal{A}$ is unital, and hence $X$ is compact, local synthesis is the same as spectral synthesis. We say that $E$ is approximable for $\mathcal{A}$ if $\mathrm{I}_{\mathcal{A}}(E)$ admits a bounded approximate identity. We say $E$ is essential for $\mathcal{A}$ if $\mathrm{I}_{\mathcal{A}}(E)$ is an essential module over itself, i.e. $\overline{\mathrm{I}_{\mathcal{A}}(E)^{2}}=\mathrm{I}_{\mathcal{A}}(E)$.

Let us summarise, for convenience of the reader, some of the strong connections which exist between the amenability-like properties, listed above, and properties of ideals.

Lemma 1.1. Let $\mathcal{A}$ be a regular (completely contractive) Banach function algebra on a locally compact space $X$, such that $\mathcal{A} \otimes^{\gamma} \mathcal{A}$ (resp. $\mathcal{A} \widehat{\otimes} \mathcal{A}$ ) is semi-simple. Then the following conditions hold.

(i) $\mathcal{A} \otimes^{\gamma} \mathcal{A}$ (resp. $\left.\mathcal{A} \widehat{\otimes} \mathcal{A}\right)$ is regular on $X \times X$.

(ii) The product map $m_{\mathcal{A}}: \mathcal{A} \otimes^{\gamma} \mathcal{A} \rightarrow \mathcal{A}$ (resp. $m_{\mathcal{A}}: \mathcal{A} \widehat{\otimes} \mathcal{A} \rightarrow \mathcal{A}$ ) satisfies ker $m_{\mathcal{A}}=\mathrm{I}_{\mathcal{A} \otimes \gamma \mathcal{A}}(\Delta)$ (resp. ker $m_{\mathcal{A}}=\mathrm{I}_{\mathcal{A} \widehat{\otimes} \mathcal{A}}(\Delta)$ ) where $\Delta=\{(x, x): x \in X\}$.

(iii) $\mathcal{A}$ is (operator) amenable if and only if $\mathcal{A}$ has a bounded approximate identity and $\Delta$ is approximable for $\mathcal{A} \otimes^{\gamma} \mathcal{A}$ (resp. $\left.\mathcal{A} \widehat{\otimes} \mathcal{A}\right)$.

(iv) $\mathcal{A}$ is (operator) hyper-Tauberian on $X$ if and only if $\mathcal{A}$ is Tauberian and $\Delta$ is a set of local synthesis for $\mathcal{A} \otimes^{\gamma} \mathcal{A}$ (resp. $\left.\mathcal{A} \widehat{\otimes} \mathcal{A}\right)$.

(v) If $\mathcal{A}$ has a bounded approximate identity, then $\mathcal{A}$ is (operator) weakly amenable if and only if $\overline{\mathcal{A}^{2}}=\mathcal{A}$ and $\Delta$ is essential for $\mathcal{A} \otimes^{\gamma} \mathcal{A}$ (resp. $\left.\mathcal{A} \widehat{\otimes} \mathcal{A}\right)$.

Proof. (i) This is [35, Theorem 3]. The semisimplicity of $\mathcal{A} \otimes^{\gamma} \mathcal{A}$ (resp. $\mathcal{A} \widehat{\otimes} \mathcal{A})$ is sufficient to guarantee that it is a function algebra on $X \times X$. 
(ii) This is immediate.

(iii) This follows from (i) and a splitting result of [24] (see also [3, Theorem $3.10]$ ). The proof of [3, Theorem 3.10] can be adapted work in the completely contractive case, see [36, Theorem 3] or [11, Lemma 2.1].

(iv) This is [32, Theorem 6]. It is noted in the proof of [10, Thoerem 3.10] how to adapt this to the operator space setting. We note that these proofs work for our general assumptions so we do not need that $X$ is the spectrum of $\mathcal{A}$.

(v) This follows fom (ii) and [13, Theorem 3.2] (see [33, Section 2] for the completely bounded version).

1.3. The Fourier algebra and some of its algebras. The Fourier algebra $\mathrm{A}(G)$, for any locally compact group, was defined in [6]. $\mathrm{A}(G)$ gains it operator space structure from being the predual of a von Neumann algebra; see [6. (3.10)] and then [5. Section 3.2]. We note that $\mathrm{A}(G) \otimes^{\gamma} \mathrm{A}(G)$ is naturally isomorphic to $\mathrm{A}(G \times G)$ only when $G$ has an abelian subgroup of finite index, by [25]. However, $\mathrm{A}(G) \widehat{\otimes} \mathrm{A}(G) \cong \mathrm{A}(G \times G)$ completely isometrically, via the natural identification; see [5, Theorem 7.2.4].

If $G$ is a compact group we let $\Delta=\{(s, s): s \in G\}$ denote the diagonal subgroup of $G \times G$, and let

$$
\mathrm{A}(G \times G: \Delta)=\{u \in \mathrm{A}(G \times G): r \cdot u=u \text { for } r \text { in } G\}
$$

where $r \cdot u(s, t)=u($ srtr $)$. Then $\mathrm{A}(G \times G: \Delta)$ is the closed subspace consists of $\mathrm{A}(G \times G)$ which of functions constant on left cosets of $\Delta$. There is a homeomorphism of the left coset space $G \times G / \Delta$ with $G$ given by $(s, t) \Delta \mapsto$ $s t^{-1}$. We let

$$
\mathrm{A}_{\Delta}(G)=\{u \text { in } \mathcal{C}(G): u(s)=w(s, e) \text { for some } w \text { in } \mathrm{A}(G \times G: \Delta)\}
$$

The map

$$
\Gamma: \mathrm{A}(G \times G) \rightarrow \mathrm{A}_{\Delta}(G), \quad \Gamma w(s)=\int_{G} w(s t, t) d t
$$

is surjective, and is injective on $\mathrm{A}(G \times G: \Delta)$. We endow $\mathrm{A}_{\Delta}(G)$ with the norm and operator space structure which make $\Gamma$ a complete quotient map. We note then that

$$
N: \mathrm{A}_{\Delta}(G) \rightarrow \mathrm{A}(G \times G: \Delta), \quad N u(s, t)=u\left(s t^{-1}\right)
$$

is thus a complete isometry. We note that $\mathrm{A}_{\Delta}(G) \widehat{\otimes} \mathrm{A}_{\Delta}(G) \cong \mathrm{A}_{\Delta}(G \times G)$ by [10. Proposition 2.5]. If we repeat the procedure above we obtain

$$
\mathrm{A}_{\Delta^{2}}(G)=\Gamma\left(\mathrm{A}_{\Delta}(G \times G)\right) .
$$

We can do a similar construction with $\check{\Delta}=\left\{\left(s, s^{-1}\right): s \in G\right\}$. We let $G \times$ $G / \check{\Delta}$ denote the set of equivalence classes modulo the equivalence relation $\left(s^{\prime}, t^{\prime}\right) \sim(s, t)$ if and only if $\left(s^{\prime} s^{-1}, t^{-1} t^{\prime}\right) \in \check{\Delta}$, so $G \times G / \check{\Delta}$, with the quotient topology, is homeomorphic to $G$ via $(s, t) \check{\Delta} \mapsto s t$. We let

$$
\mathrm{A}(G \times G: \check{\Delta})=\{u \in \mathrm{A}(G \times G): r \diamond u=u \text { for } r \text { in } G\}
$$


where $r \diamond u(s, t)=u\left(s r, r^{-1} t\right)$. Similarly as above, $\mathrm{A}(G \times G: \check{\Delta})$ is a closed subalgebra of $\mathrm{A}(G \times G)$. Let

$$
\mathrm{A}_{\gamma}(G)=\{u \text { in } \mathcal{C}(G): u(s)=w(s, e) \text { for some } w \text { in } \mathrm{A}(G \times G: \check{\Delta})\}
$$

Then the map

$$
\check{\Gamma}: \mathrm{A}(G \times G) \rightarrow \mathrm{A}_{\gamma}(G), \quad \Gamma w(s)=\int_{G} w\left(s t, t^{-1}\right) d t
$$

is surjective, and is injective on $\mathrm{A}(G \times G: \check{\Delta})$. We endow $\mathrm{A}_{\gamma}(G)$ with the norm and operator space structure which make $\check{\Gamma}$ a complete quotient map. We also note that

$$
\check{N}: \mathrm{A}_{\gamma}(G) \rightarrow \mathrm{A}(G \times G: \check{\Delta}), \quad N u(s, t)=u(s t)
$$

is a complete isometry.

We note the origional construction of $\mathrm{A}_{\gamma}(G)$, from [22]. Let us first observe that $\mathrm{A}(G)$ has the approximation property, being metrically an $\ell^{1}$ direct sum of finite dimensional trace class algebras [18, (34.4)], and thus $\mathrm{A}(G) \otimes^{\gamma} \mathrm{A}(G)$ is semi-simple with spectrum $G \times G$, by [35, Theorem 4]. Thus $\mathrm{A}(G) \otimes^{\gamma} \mathrm{A}(G)$ can be regarded as a subalgebra of $\mathrm{A}(G \times G)$, and we can restrict the maps $\Gamma$ and $\check{\Gamma}$ to $\mathrm{A}(G) \otimes^{\gamma} \mathrm{A}(G)$. We have

$$
\Gamma\left(\mathrm{A}(G) \otimes^{\gamma} \mathrm{A}(G)\right)=\check{\Gamma}\left(\mathrm{A}(G) \otimes^{\gamma} \mathrm{A}(G)\right)=\mathrm{A}_{\gamma}(G)
$$

as was observed in [10, Section 4.2]. Moreover, each of $\Gamma, \check{\Gamma}: \mathrm{A}(G) \otimes^{\gamma} \mathrm{A}(G) \rightarrow$ $\mathrm{A}_{\gamma}(G)$ are quotient maps. In $\left[22, \mathrm{~A}_{\gamma}(G)\right.$ is used in a careful and clever way to study the amenability properties of $\mathrm{A}(G)$. It is curious that the projective, and operator projective products of $\mathrm{A}(G)$ produce the same result, here.

It is easily shown (see [10, Section $2.1 \&$ Section 4.2]) that

$$
\mathrm{A}_{\Delta^{2}}(G) \subset \mathrm{A}_{\gamma}(G) \subset \mathrm{A}_{\Delta}(G) \subset \mathrm{A}(G) .
$$

with equality holding for any pair, and hence all pairs, exactly when $G$ has an open abelian subgroup. Moreover, the inclusion map are all contractions.

We note that each of $\mathrm{A}(G)$ and $\mathrm{A}_{\Delta}(G)$ have spectrum $G$ - see [6] and [10, Proposition 1.1] respectively - and $\mathrm{A}(G)$ is Tauberian. Since $\mathrm{A}(G \times G)$ is regular on $G \times G$, it follows that each of $\mathrm{A}_{\Delta}(G)$ and $\mathrm{A}_{\gamma}(G)$ is regular on $G$; inductively it follows that $\mathrm{A}_{\Delta^{2}}(G)$ is regular on $G$. For example, if $K$ and $L$ are disjoint compact subsets of $G$, then $K^{*}$ and $L^{*}$ are disjoint compact subsets of $G \times G$ where $E^{*}=\left\{(s, t) \in G \times G: s t^{-1} \in E\right\}$ for $E \subset G$. Find $w \in \mathrm{A}(G \times G)$ such that $\left.w\right|_{K^{*}}=1$ and $\left.w\right|_{L^{*}}=0$. Then $u=\Gamma w$ satisfies $\left.u\right|_{K}=1$ and $\left.u\right|_{L}=0$, showing that $\mathrm{A}_{\Delta}(G)$ is regular on $G$. Though we suspect it to be true, we have not verified that either $\mathrm{A}_{\gamma}(G)$ or $\mathrm{A}_{\Delta^{2}}(G)$ have spectrum $G$.

\section{Compact groups}

2.1. Weak amenability of Fourier algebras. We let $G_{e}$ denote the connected component of the identity in $G$. The following result, for compact groups, is the the converse of [9, Theorem 3.3]. 
Theorem 2.1. Let $G$ be a compact group. Then the following are equivalent:

(i) $\mathrm{A}(G)$ is weakly amenable;

(ii) $\mathrm{A}(G)$ is hyper-Tauberian;

(iii) $G_{e}$ is abelian.

Proof. That (iii) implies (ii) is [32, Theorem 21]. That (ii) implies (i) is [32, Theorem 5]. (We note that (iii) implies (i) follows from [9, Theorem 3.3].) Thus it suffices to show that (i) implies (iii).

The restriction map $\left.u \mapsto u\right|_{G_{e}}$ from $\mathrm{A}(G)$ to $\mathrm{A}\left(G_{e}\right)$ is a surjection by [16]. Thus, by [4][Proposition 2.8.64], if $\mathrm{A}(G)$ is weakly amenable, then so too must be $\mathrm{A}\left(G_{e}\right)$. Hence we assume that $G$ is connected, so $G=G_{e}$.

Suppose $G$ is connected and nonabelian. Then by [28, Theorem 6.5.6] there is a family $\left\{G_{i}\right\}_{i \in I}$ of compact connected Lie groups, at least one of which is simple (in the sense of Lie groups) such that

$$
G \cong\left(\prod_{i \in I} G_{i}\right) / A
$$

where $A$ is a closed subgroup of the centre of $P=\prod_{i \in I} G_{i}$. Let $G_{i_{0}}$ be simple. Then, as shown in [27], there is a closed subgroup $K$ of $G_{i_{0}}$ such that

$$
\text { either } K \cong \mathrm{SU}(2) \text { or } K \cong \mathrm{SO}(3) \text {. }
$$

Let $H=K A / A$, where $K$ is embedded into $P$ in the natural way. By the second isomorphism theorem, $H \cong K /(K \cap A)$. We have $K \cap A \subset \mathrm{Z}(K)$, the centre of $K$. We note that $\mathrm{Z}(\mathrm{SO}(3))$ is trivial, while $\mathrm{Z}(\mathrm{SU}(2))=\{-1,1\}$ and $\mathrm{SU}(2) /\{-1,1\} \cong \mathrm{SO}(3)$. It follows that $H$ is isomorphic to one of $\mathrm{SO}(3)$ or $\mathrm{SU}(2)$. In either case, it follows from [27] (via [22, Corollary 7.3]) that $\mathrm{A}(H)$ is not weakly amenable. Since $H$ is isomorphic to a closed subgroup of $G$, the restriction map gives a homomorphism from $\mathrm{A}(G)$ onto $\mathrm{A}(H)$. Hence $\mathrm{A}(G)$ can not be weakly amenable either.

Thus we conclude that for $\mathrm{A}(G)$ to be weakly amenable, $G_{e}$ must be abelian.

Theorem 2.2. For a compact group $G$, if $\mathrm{A}(G)$ contains a weakly amenable non-zero closed ideal, then $G_{e}$ is abelian.

Proof. Let $\mathcal{I}$ be a non-zero closed ideal, and

$$
E=\operatorname{hull}(\mathcal{I})=\{s \in G: u(s)=0 \text { for every } u \text { in } \mathcal{I}\} .
$$

Suppose $\mathcal{I}$ is weakly amenable. Since left translation $u \mapsto s * u(s * u(t)=$ $\left.u\left(s^{-1} t\right)\right)$ is an automorphism of $\mathrm{A}(G)$, for each $s$ in $G$, each $s * \mathcal{I}$ is an ideal with hull $(s * \mathcal{I})=s E$. For each finite subset $F$ of $G$, the ideal $\mathcal{I}_{F}=\overline{\sum_{s \in F} s * \mathcal{I}}$ is weakly amenable. Indeed, $\mathcal{I}_{F}^{*}$ is a symmetric module over each $s * \mathcal{I}$, so by [2. Theorem 1.5] any bounded derivation $D: \mathcal{I}_{F} \rightarrow \mathcal{I}_{F}^{*}$ must satisfy $\left.D\right|_{s * \mathcal{I}}=$ 0 for each $s$ in $F$. Moreover, $\operatorname{hull}\left(\mathcal{I}_{F}\right)=\cap_{s \in F} s E$. By finite intersection property we have either that

(a) $\cap_{s \in F} s E=\varnothing$ for some $F$, or 
(b) $\cap_{s \in G} s E \neq \varnothing$.

In case (b), there is some $t \in \cap_{s \in G} s E$, and thus $s \in E t^{-1}$ for every $s$ in $G$, which implies that $E=G$. However, $\operatorname{hull}(\mathcal{I})=G$, only for $\mathcal{I}=\{0\}$, contradicting assumptions. Hence case (a) holds, and for such $F, \mathcal{I}_{F}=$ $\mathrm{A}(G)$, since $\mathrm{A}(G)$ is Tauberian. But then $\mathrm{A}(G)$ itself is weakly amenable, and $G_{e}$ is abelian by Theorem 2.1 .

2.2. Spectral synthesis of the anti-diagonal. Let $G$ be a compact group.

Lemma 2.3. Let $\theta: G \times G \rightarrow G$ be given by $\theta(s, t)=s t^{-1}$, and $\check{\theta}: G \times G \rightarrow$ $G$ be given by $\theta(s, t)=$ st. Then for a closed subset $E$ of $G$, the following are equivalent:

(i) $E$ is spectral [approximable/essential] for $\mathrm{A}_{\gamma}(G)$;

(ii) $\check{\theta}^{-1}(E)$ is spectral [approximable/essential] for $\mathrm{A}(G \times G)$;

(iii) $\theta^{-1}(E)$ is spectral [approximable/essential] for $\mathrm{A}(G) \otimes^{\gamma} \mathrm{A}(G)$;

(iii') $\check{\theta}^{-1}(E)$ is spectral [approximable/essential] for $\mathrm{A}(G) \otimes^{\gamma} \mathrm{A}(G)$.

Proof. The equivalence of (i) and (iii) follows immediately from [10, Corollary 1.5$]$.

The equivalence of (i) and (ii), respectively of (i) and (iii'), follows similarly, but a few modifications are required. In [10, Theorem 1.4] we implicitly used the group action of $G$ on $\mathrm{A}(G \times G)$, respectively on $\mathrm{A}(G) \otimes \otimes^{\gamma} \mathrm{A}(G)$, given by $(r, w) \mapsto r \cdot w$ (where $r \cdot w(s, t)=w(s r, t r)$ ); see [34, Theorem 3.1] to see this action used more explicitly. If the above group action is replaced by $(r, w) \mapsto r \diamond w$ (where $r \diamond w(s, t)=w\left(s r, r^{-1} t\right)$ ), then it can be shown, similarly to [10, Theorem 1.4] or [34, Theorem 3.1], that

$$
\check{\Gamma} \mathrm{I}_{\mathcal{A}(G \times G)}\left(\check{\theta}^{-1}(E)\right)=\mathrm{I}_{\mathrm{A}(G)}(E) \quad \text { and } \quad\left\langle\check{N} \mathrm{I}_{\mathrm{A}(G)}(E)\right\rangle=\mathrm{I}_{\mathcal{A}(G \times G)}\left(\check{\theta}^{-1}(E)\right)
$$

where $\mathcal{A}(G \times G)$ is $\mathrm{A}(G \times G)$, respectively $\mathrm{A}(G) \otimes \otimes^{\gamma} \mathrm{A}(G)$, and $\langle\mathcal{S}\rangle$ denotes the closed ideal generated by $\mathcal{S}$. The desired results then can be proved exactly as in [10, Corollary 1.5].

In [9] it is shown that $\mathrm{A}(G)$ is amenable exactly when $\check{\Delta}=\left\{\left(s, s^{-1}\right)\right.$ : $s \in G\}$ is an element of the coset ring of $G \times G$, exactly when $G$ admits an abelian subgroup of finite index. The next result is analogous to that.

Theorem 2.4. Let $G$ be a compact group. Then the following are equivalent:

(i) $\mathrm{A}(G)$ is weakly amenable;

(ii) $\check{\Delta}$ is spectral for $\mathrm{A}(G \times G)$;

(iii) $\check{\Delta}$ is essential for $\mathrm{A}(G \times G)$;

(iv) $G_{e}$ is abelian.

Proof. The equivalence of (i) and (iv) is established in Theorem 2.1, That (ii) implies (iii) is clear.

If (iii) holds, then $\check{\theta}(\check{\Delta})=\{e\}$ is essential for $\mathrm{A}_{\gamma}(G)$ by Lemma 2.3, (iii) implies (i). It then follows, as in the proof of [22, Theorem 7.2], that $\mathrm{A}(G)$ is weakly amenable. 
If (iv) holds, then $\mathrm{A}(G)$ is hyper-Tauberian by [32, Theorem 21]. But then by [32, Theorem 6] $\Delta$ is a set of local synthesis for $\mathrm{A}(G) \otimes^{\gamma} \mathrm{A}(G)$, hence a set of synthesis as $G$ is compact. Thus by Lemma 2.3, (iii) implies (i), $\{e\}$ is a set of synthesis for $\mathrm{A}_{\gamma}(G)$. But by Lemma 2.3. (i) implies (ii), we get that $\check{\Delta}$ is spectral for $\mathrm{A}(G \times G)$.

We finish this section by giving alternative proofs to some results from [22]. While our proof is ultimately no more efficient than the proofs given there, it places the results in a more general framework.

Proposition 2.5. Let $G$ be a compact group. Then

(i) [22, Theorem 3.2] $\mathrm{A}(G)$ is amenable if and only if $\mathrm{I}_{\mathrm{A}_{\gamma}(G)}\{e\}$ has a bounded approximate identity; and

(ii) [22, Theorem 7.2] $\mathrm{A}(G)$ is weakly amenable if and only if $\overline{\mathrm{I}_{\mathrm{A}_{\gamma}(G)}\{e\}^{2}}=$ $\mathrm{I}_{\mathrm{A}_{\gamma}(G)}\{e\}$.

Proof. These are immediate consequences of the equivalence of (i) and (iii) in Lemma 2.3, and Lemma 1.1, (iii) and (v), respectively.

2.3. The connection between cohomology of $\mathrm{A}(G)$ and cohomology of $\mathrm{A}_{\Delta}(G)$. We illustrate, in the next two theorems, a curious connection that exists between cohomology of $\mathrm{A}(G)$, operator cohomology of $\mathrm{A}_{\Delta}(G)$ and cohomology of $\mathrm{A}_{\Delta}(G)$.

Theorem 2.6. Let $G$ be a compact group. Then the following are equivalent:

(i) $\mathrm{A}_{\Delta}(G)$ is operator weakly amenable;

(i') $\mathrm{A}_{\Delta}(G)$ is weakly amenable;

(ii) $\mathrm{A}_{\Delta}(G)$ is operator hyper-Tauberian;

(ii') $\mathrm{A}_{\Delta}(G)$ is hyper-Tauberian;

(iii) $G_{e}$ is abelian.

Proof. That (i') implies (i), and (ii') implies (ii) are clear. That (ii') implies (i') is from [32, Theorem 5], and the completely bounded analogue, that (ii) implies (i), is noted in [32, Theorem 26]. That (iii) implies (ii') is from [10, Theorem 3.7] and the identification $\mathrm{A}_{\Delta}(G)=\mathrm{A}(G \times G: \Delta)$. Thus it remains to show that (i) implies (iii).

If $\mathrm{A}_{\Delta}(G)$ is operator weakly amenable, then since $\mathrm{A}_{\Delta}(G) \widehat{\otimes} \mathrm{A}_{\Delta}(G) \cong$ $\mathrm{A}_{\Delta}(G \times G)$ by [10, Proposition 2.5 (i)], it follows Lemma 1.1] that

$$
\overline{\mathrm{I}_{\mathrm{A}_{\Delta^{2}}(G)}\{e\}^{2}}=\mathrm{I}_{\mathrm{A}_{\Delta^{2}}(G)}\{e\} .
$$

We will show that

$$
\overline{\mathrm{I}_{\mathrm{A}_{\gamma}(G)}\{e\}^{2}}=\mathrm{I}_{\mathrm{A}_{\gamma}(G)}\{e\} .
$$

Indeed, let us first note that

$$
\mathrm{I}_{\mathrm{A}_{\Delta^{2}}(G)}\{e\} \text { is dense in } \mathrm{I}_{\mathrm{A}_{\gamma}(G)}\{e\} .
$$


Since $\mathrm{A}_{\Delta^{2}}(G)$ is dense in $\mathrm{A}_{\gamma}(G)$, for any $u$ in $\mathrm{I}_{\mathrm{A}_{\gamma}(G)}\{e\}$ there is a sequence $\left(u_{n}\right) \subset \mathrm{A}_{\Delta^{2}}(G)$ which converges to $u$. We note that

$$
\left|u_{n}(e)\right|=\left|u_{n}(e)-u(e)\right| \leq\left\|u_{n}-u\right\|_{\infty} \leq\left\|u_{n}-u\right\|_{\mathrm{A}_{\gamma}} \stackrel{n \rightarrow \infty}{\longrightarrow} 0 .
$$

Thus if $u_{n}^{\prime}=u_{n}-u_{n}(e) 1$, then $\left(u_{n}^{\prime}\right) \subset \mathrm{I}_{\mathrm{A}_{\Delta^{2}}(G)}\{e\}$ with $\lim _{n \rightarrow \infty}\left\|u_{n}^{\prime}-u\right\|_{\mathrm{A}_{\gamma}}=$ 0 . Then (2.2) follows form (2.1) and (2.3).

By Proposition 2.5 (ii), (2.2) implies that $\mathrm{A}(G)$ is weakly amenable. Thus it follows from Theorem 2.1 that $G_{e}$ is abelian.

Theorem 2.7. Let $G$ be a compact group. Then the following are equivalent:

(i) $\mathrm{A}_{\Delta}(G)$ is operator amenable;

(i') $\mathrm{A}_{\Delta}(G)$ is amenable;

(ii) $\mathrm{A}(G)$ is amenable;

(iii) $G$ admits an open abelian subgroup.

Proof. Clearly (i') implies (i). The equivalence of (ii) and (iii) is [9, Theorem 2.3]. By [10, Corollary 2.4], if (iii) holds then $\mathrm{A}(G)=\mathrm{A}_{\Delta}(G)$, isomorphically, and hence (i') holds. Thus it suffices to show that (i) implies (ii).

If (i) holds, then from Lemma 1.1 (iii) we have that $\mathrm{I}_{\mathrm{A}_{\Delta}(G \times G)}(\Delta)$ has a bounded approximate identity. But then it follows from [10, Corollary 1.5 (i)] that $\mathrm{I}_{\mathrm{A}_{\triangle 2}(G)}\{e\}$ has a bounded approximate identity. From (2.3) it follows that $\mathrm{I}_{\mathrm{A}_{\gamma}(G)}\{e\}$ has a bounded approximate identity. Then by Proposition $2.5(\mathrm{i}), \mathrm{A}(G)$ is amenable.

Comparing Theorems 2.6 and 2.7, it seems that the operator cohomology of $\mathrm{A}_{\Delta}(G)$ is trivial exactly when the same is true of the cohomology of $\mathrm{A}_{\Delta}(G)$, which, in turn, is the same as the vanishing of the the cohomology of $\mathrm{A}(G)$. This leads to a natural question.

Is the operator space structure on $\mathrm{A}_{\Delta}(G)$ maximal?

A positive answer to the above question would explain many of the implications if Theorems 2.6 and 2.7. Though it seems unlikely that the operator space structure on $\mathrm{A}_{\Delta}(G)$ is maximal, in general, there is further curiosity related to this, which we indicate below.

As shown in [9, Proposition 1.5], the map $u \mapsto \check{u}: \mathrm{A}(G) \rightarrow \mathrm{A}(G)$ (where $\left.\check{u}(s)=u\left(s^{-1}\right)\right)$ is completely bounded only when $G$ has an abelian subgroup of finite index. By [12, Theorem 4.5], this is equivalent to $\mathrm{A}(G)$ having the maximal operator space structure. However, this map is a complete isometry only when $G$ is abelian; see [19, Proposition 3.1] and [30, Proposition 3.4].

Proposition 2.8. For any compact group $G$, the map $u \mapsto \check{u}$ is a complete isometry on $\mathrm{A}_{\Delta}(G)$.

Proof. The map $\sigma: G \times G \rightarrow G \times G$ given by $\sigma(s, t)=(t, s)$ is an isomorphism, hence $J_{\sigma}: \mathrm{A}(G \times G) \rightarrow \mathrm{A}(G \times G), J_{\sigma} w=w \circ \sigma$, is a completely isometric homomorphism; see, for example, [19, Proposition 3.1]. It is clear that $J_{\sigma}(\mathrm{A}(G \times G: \Delta))=\mathrm{A}(G \times G: \Delta)$. Let $N$ be as in (1.1), so $N$ has inverse 
$M: \mathrm{A}(G \times G: \Delta) \rightarrow \mathrm{A}_{\Delta}(G)$ given by $M w(s)=w(s, e)$, which is thus also a complete isometry. We note that

$$
\left[M J_{\sigma} N u\right](s)=\left[J_{\sigma} N u\right](s, e)=N u(e, s) \stackrel{\dagger}{=} N u\left(s^{-1}, e\right)=u\left(s^{-1}\right)=\check{u}(s)
$$

where we used that $N u \in \mathrm{A}(G \times G: \Delta)$ at $\dagger$. Hence $u \mapsto \check{u}$, which factors as three complete contractions, is itself a complete contraction. This map is obviously its own inverse, hence is a complete isometry.

\section{SOME NON-COMPACT GROUPS}

\subsection{A general weak amenability result.}

Theorem 3.1. If $G$ is a locally compact group for which $\mathrm{A}(G)$ admits a non-zero weakly amenable ideal, then $G$ contains no non-abelian compact connected subgroups. In particular, this holds for $\mathcal{I}=\mathrm{A}(G)$.

Proof. Let $\mathcal{I}$ be a non-zero ideal of $\mathrm{A}(G)$, and $K$ be a connected compact subgroup of $G$. Since $E=\operatorname{hull}(\mathcal{I}) \neq G$, there is $s$ in $G$ such that $s E \not \supset$ $K$, and $s * \mathcal{I}$ is also a weakly amenable ideal. Since the restriction map $\left.u \mapsto u\right|_{K}: \mathrm{A}(G) \rightarrow \mathrm{A}(K)$ is surjective, by [16], $\mathcal{J}=\overline{\left.s * \mathcal{I}\right|_{K}}$ is a closed ideal of $\mathrm{A}(K)$. The choice of $s$ ensures that hull $(\mathcal{J}) \neq K$. Since $\mathcal{J}$ is the closure of the image of a weakly amenable algebra, $\mathcal{J}$ is weakly amenable by [4, Proposition 2.8.64]. It then follows from Theorem 2.2 that $K$ must be abelian.

The above result while very broad, misses still some key examples such as $\mathrm{SL}_{2}(\mathbb{R})$, the $a x+b$-group and the Heisenberg group. As we mentioned before, it is conjectured that $\mathrm{A}(G)$ can be weakly amenable only when $G_{e}$ is abelian. It may well be the case, that $G_{e}$ is abelian if $\mathrm{A}(G)$ has even a non-zero weakly amenable closed ideal.

3.2. Local synthesis of the anti-diagonal. We begin with a lemma, indicating a local property of local synthesis.

Lemma 3.2. Let $\mathcal{A}$ be a regular semi-simple commutative Banach algebra with spectrum $\Omega$, and $E$ be a closed subset of $\Omega$. If each point in $E$ admits a closed neighbourhood $N$ such that $N \cap E$ is of local synthesis, then $E$ itself is of local synthesis.

Proof. Let $u \in \mathrm{I}^{c}(E)$. By assumption, there exists, for each $x \in E$, a closed neighbourhood $N_{x}$ of $x$ such that

$$
u \in \overline{\mathrm{I}^{0}\left(E_{x}\right)} \text { where } E_{x}=N_{x} \cap E
$$

as $\mathrm{I}^{c}\left(E_{x}\right) \supset \mathrm{I}^{c}(E) \ni u$. By regularity of $\mathcal{A}$, there is $h_{x} \in \mathcal{A}$ whose support is contained in the interior of $N_{x}$ and for which there is an open neighbourhood $U_{x}$ of $x$ such that $\left.h_{x}\right|_{U_{x}}=1$. By (3.1), given $\varepsilon>0$, there is $u_{x} \in \mathrm{I}^{0}\left(E_{x}\right)$ such that $\left\|u-u_{x}\right\|<\varepsilon /\left\|h_{x}\right\|$. Hence it follows that $\left\|h_{x} u-h_{x} u_{x}\right\|<\varepsilon$. We have that $h_{x} u_{x} \in \mathrm{I}^{0}(E)$ since $h_{x} u_{x}$ vanishes on a neighbourhood of $E_{x} \cup\left(\Omega \backslash N_{x}\right)$, and hence on a neighbourhood of $E$. Since $\varepsilon$ is arbitrary, we have that 
$h_{x} u \in \overline{\mathrm{I}^{0}(E)}$ and $\left.h_{x} u_{x}\right|_{U_{x}}=\left.u_{x}\right|_{U_{x}}$. In other words, " $u$ is locally contained in $\overline{\mathrm{I}^{0}(E)}$ ". Then, a standard partition of unity argument shows that $u \in \overline{\mathrm{I}^{0}(E)}$.

With the above lemma we can obtain The following generalisation of Theorem 2.4.

Theorem 3.3. Let $G$ be a locally compact group which admits an open subgroup $H \cong A \times K$, where $A$ is an abelian group and $K$ is compact. Then the following are equivalent:

(i) $\mathrm{A}(G)$ is weakly amenable;

(ii) $\mathrm{A}(G)$ is hyper-Tauberian;

(iii) $\check{\Delta}=\left\{\left(s, s^{-1}\right): s \in G\right\}$ is a set of local synthesis for $\mathrm{A}(G \times G)$;

(iv) $\mathrm{I}_{\mathrm{A}(G \times G)}^{\prime}(\check{\Delta})=\overline{\mathrm{A}(G \times G) \mathrm{I}_{\mathrm{A}(G \times G)}(\check{\Delta})}$ is operator weakly amenable;

(v) $G_{e}$ is abelian.

Proof. That (v) implies (ii) is [32, Theorem 21], and that (ii) implies (i) is [32, Theorem 5]. If (i) holds, then by Theorem [3.1, $K_{e}$ is abelian, hence $G_{e}=H_{e} \cong A \times K_{e}$ is abelian, so (v) holds.

Suppose (v) holds. Then $K_{e}$ is abelian, and hence by Theorem $2.4 \check{\Delta}_{K}$ is a set of synthesis for $\mathrm{A}(K \times K)$. Since $\check{\Delta}_{A}$ is a subgroup of $A \times A$, it is a set of synthesis for $\mathrm{A}(A \times A)$ by [16, Theorem 2]. Hence it follows (the operator space analogue of) [23, Theorem 1.6], that $\check{\Delta}_{A} \times \check{\Delta}_{K}$ is a set of synthesis for $\mathrm{A}(A \times A) \widehat{\otimes} \mathrm{A}(K \times K) \cong \mathrm{A}(A \times A \times K \times K)$. By applying a suitable automorphism it follows that $\check{\Delta}_{H} \cong \check{\Delta}_{A \times K}$ is a set of synthesis for $\mathrm{A}(H \times H) \cong \mathrm{A}(A \times K \times A \times K)$. Hence $\check{\Delta}_{H}$ is a set of synthesis for $\mathrm{A}(G \times G)$, since $H$ is open in $G$. If $\left(s, s^{-1}\right) \in \check{\Delta}_{G}$, then $s=t h$ for some $t$ in $G$ and $h$ in $H$. Hence $\left(s, s^{-1}\right) \in(t, e) \check{\Delta}_{H}\left(e, t^{-1}\right)$. Letting $N_{s}=(t, e) H \times H\left(e, t^{-1}\right)$ we have that $\check{\Delta}_{G} \cap N_{s}=(t, e) \check{\Delta}_{H}\left(e, t^{-1}\right)$ is a set of (local) synthesis. Hence by Lemma 3.2 we obtain (iii).

If (iii) holds, then

$$
\mathrm{I}_{\mathrm{A}(G \times G)}^{\prime}(\check{\Delta})=\overline{\mathrm{I}_{\mathrm{A}(G \times G)}^{c}(\check{\Delta})}=\mathrm{I}_{\mathrm{A}(G \times G)}^{0}(\check{\Delta})
$$

by regularity of $G$, and thus $\mathrm{I}_{\mathrm{A}(G \times G)}^{\prime}(\check{\Delta})$ is essential. Since $\mathrm{A}(G \times G)$ is operator weakly amenable by [33], it follows [12, Proposition 3.5] (which is the completely bounded version of [14, Corollary 1.3]) that (iv) holds.

Finally, suppose (iv) holds. Then $\left.\mathrm{I}_{\mathrm{A}(G \times G)}^{\prime}\left(\check{\Delta}_{G}\right)\right|_{K \times K}=\mathrm{I}_{\mathrm{A}(K \times K)}\left(\check{\Delta}_{K}\right)$ is operator weakly amenable. Hence $\mathrm{I}_{\mathrm{A}(K \times K)}\left(\check{\Delta}_{K}\right)$ is essential by [12, Lemma 3.1]. Thus by Lemma 2.3 (ii) with $E=\{e\}$, and Proposition 2.5 (ii), $\mathrm{A}(K)$ is weakly amenable. Hence $K_{e}$ is abelian and so $G_{e} \cong A_{e} \times K_{e}$ is abelian, and we obtain $(\mathrm{v})$.

We say that a locally compact group $G$ has small invariant neighbourhoods if there is a basis for the identity of neighbourhoods invariant under inner automorphisms. We say $G$ is maximally almost periodic if it continuously embeds into a compact group. 
Corollary 3.4. If $G$ is either a small invariant neighbourhood group, or a maximally almost periodic group, then the equivalent conditions of Theorem 3.3 hold.

Proof. Any locally compact group $G$ admits an open almost connected subgroup $H$ by [17, (7.3) \& (7.7)]. If $G$ is either a small invariant neighbourhood group, or is maximally almost periodic, clearly $H$ has the same property. Then by [15, Theorem 2.9] $H$ is isomorphic to a semi-direct product $V \ltimes_{\eta} K$ where $V$ is a vector group and $K$ is compact with $\eta(K)$ a finite subgroup of automorphisms on $V$. (See [26, pages 1486-1487].) Then $V \times \operatorname{ker} \eta$ is an open subgroup of $H$, and hence of $G$. Thus we may appeal directly to Theorem 3.3 .

\section{REFERENCES}

[1] A. Azimifard, E. Samei, and N. Spronk. Amenability properties of the centres of group algebras. Preprint, see arXiv:0805.3685 2008.

[2] W.G. Bade, P.C. Curtis, and H.G. Dales. Amenability and weak amenability for Beurling and Lipschitz algebras. Proc. London Math. Soc., 55:359-377, 1987.

[3] P.C. Curtis and R.J. Loy. The structure of amenable Banach algebras. J. London Math. Soc., 40:89-104, 1989.

[4] H.G. Dales. Banach Algebras and Automatic Continuity, volume 24 of London Math. Soc., New Series. Claredon Press, Oxford Univ. Press, New York, 2000.

[5] E.G. Effros and Z.-J. Ruan. Operator Spaces, volume 23 of London Math. Soc., New Series. Claredon Press, Oxford Univ. Press, New York, 2000.

[6] P. Eymard. L'algèbre de Fourier d'un groupe localement compact. Bull. Soc. Math. France, 92:181-236, 1964.

[7] Forrest, Brian. Amenability and Derivations of the Fourier algebra. Proc. Amer. Math. Soc., 2:437-442, 1988.

[8] Forrest, Brian. Fourier analysis on coset spaces. Rocky Mountain J. Math., 28:173190, 1998.

[9] B.E. Forrest and V. Runde. Amenability and weak amenability of the Fourier algebra. Math. Z., 250:731-744, 2005.

[10] B.E. Forrest, E. Samei, and N. Spronk. Convolutions on compact groups and Fourier algebras of coset spaces. Preprint, see arXiv:0705.4277, 2008.

[11] B.E. Forrest and N. Spronk. Best bounds for approximate identitiesin ideals of the Fouirier algebra vanishing on subgroups. Proc. Amer. Math. Soc., 134:111-116, 2005.

[12] B.E. Forrest and P.J. Wood. Cohomology and the operator space structure of the Fourier algebra and its second dual. Indiana Univ. Math. J., 50:1217-1240, 2001.

[13] N. Groenbaek. A characterization of weakly amenable Banach algebras. Studia Math., 94:149-162, 1989.

[14] N. Groenbaek. Commutative Banach algebras, module derivations and semigroups. J. London Math. Soc. (2), 40:137-157, 1989.

[15] S. Grosser and M. Moskowitz. Compactness conditions in topological groups. J. Reine Angew. Math., 246:1-40, 1971.

[16] C.S. Herz. Harmonic synthesis for subgroups. Ann. Inst. Fourier, Grenoble, 23(3):91123, 1973.

[17] E. Hewitt and K.A. Ross. Abstract Harmonic Analysis I, volume 115 of Grundlehern der mathemarischen Wissenschaften. Springer, New York, second edition, 1979.

[18] E. Hewitt and K.A. Ross. Abstract Harmonic Analysis II, volume 152 of Grundlehern der mathemarischen Wissenschaften. Springer, New York, 1970. 
[19] M. Ilie and N. Spronk. Completely bounded homomorphisms of the Fourier algebras. J. Funct. Anal., 225:480-499, 2005.

[20] B. E. Johnson. Cohomology in Banach algebras, volume 127 of Memoirs of the Amer. Math. Soc. 1972.

[21] B. E. Johnson. Weak amenability of group algebras. Bull. London Math. Soc., 23:281284, 1991.

[22] B.E. Johnson. Non-amenability of the Fourier algebra of a compact group. J. London Math. Soc., 50:361-374, 1994.

[23] E. Kaniuth. Weak spectral synthesis for the projective tensor product of commutative banach algebras. Proc. Amer. Math. Soc., 132:2959-2967, 2004.

[24] A.Ya. Khelemskii. Flat Banach modules and amenable algebras. Trudy Moskov. Mat. Obshch., 47:179-218, 1984.

[25] V. Losert. On tensor products of the Fourier algebras. Arch. Math., 43:370-372, 1984.

[26] T.W. Palmer. Banach algebras and the aeneral theory of *-algebras. Volume II. *algebras, volume 79 of Encyclopedia of Mathematics and its applications. Cambridge University Press, Cambridge, 2001.

[27] R.J. Plymen. Fourier algebra of a compact Lie group. arXiv:math.FA/0104018

[28] J. F. Price. Lie Groups and Compact Groups, volume 25 of London Math. Soc. Lec. Note Ser. Cambridge, 1977.

[29] Z.-J. Ruan. The operator amenability of $A(G)$. Amer. J. Math., 117:1449-1474, 1995.

[30] V. Runde. The amenability constant of the fourier algebra. Proc. Amer. Math. Soc., 134:1473-1481, 2006.

[31] E. Samei. Bounded and completely bounded local derivations from certain commutative semisimple Banach algebras. Proc. Amer. Math. Soc., 133:229-238, 2005.

[32] E. Samei. Hyper-Tauberian algebras and weak amenability of Figà-Talamanca-Herz algebras. J. Funct. Anal., 231:195-220, 2006.

[33] N. Spronk. Operator weak amenability of the Fourier algebra. Proc. Amer. Math. Soc., 130:3609-3617, 2002.

[34] N. Spronk and L. Turowska. Spectral synthesis and operator synthesis for compact groups. J. London Math. Soc. (2), 66:361-376, 2002.

[35] J. Tomiyama. Tensor products of commutative Banach algebras. Tôhoku Math. J., 12:143-154, 1960.

[36] P.J. Wood. Complemented idelas in the Fourier algebra of a locally compact group. Proc. Amer. Math. Soc., 128:445-451, 2000.

Address: Department of Pure Mathematics, University of Waterloo, Waterloo, ON N2L 3G1, Canada

E-mail addresses: beforres@uwaterloo.ca, esamei@uwaterloo.ca, nspronk@uwaterloo.ca 